\title{
The future of care for type 1 diabetes
}

\author{
David M. Nathan MD, Steven Russell MD PhD
}

See related research article by Haidar and colleagues on page 297 and at www.cmaj.ca/lookup/doi/10.1503/cmaj.121265

$\mathrm{T}$ ype 1 diabetes is a chronic degenerative disease characterized by gross dysregulation of glycemia, owing to autoimmune destruction of $\beta$-cell function, and by long-term complications associated with hyperglycemia. The current approach to improve the long-term course of type 1 diabetes is to try to replace insulin physiologically, with the goal of achieving blood glucose levels as close to the nondiabetic range as possible. Near-normal glycemia has been shown to reduce the development and progression of microvascular and cardiovascular disease among patients with diabetes. ${ }^{1-3}$

Two approaches to achieving near-normal glycemia have dominated our efforts in the past 3 decades. "Biologic" approaches that replace missing $\beta$-cell function by transplanting wholeorgan pancreas or isolated islets, although far more effective than in decades past, have been limited by the need for immunosuppression and its attendant risks, the risks of procedures necessary to transplant insulin-producing tissue, and the availability of organs. In addition, the limited survival of transplanted tissue, particularly isolated islets, necessitates adding exogenous insulin therapy within 2 to 4 years of transplantation. ${ }^{4}$ The "mechanical" approach has been to develop devices that emulate physiologic insulin levels. Subcutaneous administration of insulin by injection, the conventional means of insulin therapy since its introduction in 1922, suffers from delayed and erratic absorption from subcutaneous depots and its reliance on the patient to select doses to match insulin need, which is influenced by ambient glucose levels, meal size and composition, and level of activity. Given the inconstancy and hectic pace of modern life, compounded by the guesswork of insulin dosing and the inconsistent absorption, duration and peak effects of insulin with current methods of insulin therapy, it is remarkable that we have done as well as we have in managing this condition.

Although some patients with type 1 diabetes have been able to achieve glycated hemoglobin levels of less than $7 \%$, with the expectation that their long-term good health will be preserved, major challenges remain for many patients. Current intensive insulin-replacement therapy is arduous for patients, requiring frequent selfmonitoring or continuous glucose monitoring, frequent daily injections or the use of insulin pumps, and other lifestyle changes. Even when these interventions are successfully implemented, glycated hemoglobin levels are not truly normalized. Furthermore, patients who undergo intensive therapy have a 3-fold increased risk of hypoglycemia, ${ }^{1}$ which can be merely disruptive or can pose serious risks for injury or even death. Although rapid-acting insulins have reduced the rate of hypoglycemia among patients with diabetes, hypoglycemia remains one of the major impediments to intensive therapy.

Reducing the burden of intensive therapy and the frequency of hypoglycemia while maintaining near-normal glucose levels has been a major goal of treating type 1 diabetes. A promising approach is to replace decision-making by patients with a computer algorithm that receives frequent data from a continuous glucose monitor, calculates insulin dosing and automatically administers the insulin with no intervention by the patient. This apotheosis of the "mechanical" approach to insulin therapy has been called an artificial or bionic pancreas. It has the advantage of using data from continuous glucose monitors to adjust insulin dosing to the patient's changing needs, including during the vulnerable period of sleep.

Until recently, artificial pancreas systems relied only on insulin to regulate blood glucose levels. However, the normally functioning pancreas uses both insulin and glucagon to maintain glucose lev-

\section{KEY POINTS}

- Until recently, artificial pancreases relied on insulin alone to regulate blood glucose levels, leaving patients vulnerable to hypoglycemia.

- In the first randomized comparison of a dual-hormone artificial pancreas to conventional intensive therapy, Haidar and colleagues add to a growing body of evidence that glucagon can be used to prevent hypoglycemia in a dual-hormone artificial pancreas that more closely mimics normal pancreatic function.

- Insulin-only and dual-hormone approaches will need to be compared in head-to-head trials under actual home-use conditions to clarify their relative merits. 
els in the physiologic range. Glucagon opposes the effects of insulin on the liver, converting the liver from a major extractor of glucose to a net producer. Although glucagon-secreting cells are not destroyed in type 1 diabetes, the glucagon response to hypoglycemia is lost during its course, leaving patients vulnerable.

Haidar and colleagues ${ }^{5}$ add to a growing body of evidence ${ }^{6-8}$ that glucagon can be used to prevent hypoglycemia in a dual-hormone artificial pancreas that more closely mimics normal pancreatic function. Their 15-hour inpatient crossover study compared the artificial pancreas and continuous glucose monitoring with conventional pump therapy using less frequent self-monitoring.

Although their study is neither the first nor the longest investigation using a dual-hormone artificial pancreas, ${ }^{6,7}$ it is the first to compare such an apparatus to conventional intensive therapy in a randomized design. Treatment with the artificial pancreas increased the amount of time patients spent in the target range of blood glucose levels and decreased hypoglycemia. Thus, Haidar and colleagues show that low doses of glucagon administered under the control of a computer algorithm can act as a counter-regulatory hormone, preventing glucose levels from falling too low.

However, if insulin dosing algorithms were sufficiently refined, would there be any role for glucagon? Insulin sensitivity and the speed with which food is absorbed vary widely, making appropriate insulin dosing quite challenging. This problem is compounded by the slow absorption of subcutaneous insulin, even with "rapid-acting" insulin analogs, and by variable maximal effects. Finally, unanticipated exertion may reduce insulin requirements after an insulin dose has been selected by the algorithm and delivered. Any mismatch in insulin requirement and delivery owing to these factors could lead to hypoglycemia. Even complete suspension of insulin delivery is unlikely to stave off impending hypoglycemia, because it cannot erase the delayed effect of insulin already in the subcutaneous tissue but not yet absorbed into the blood. These factors likely account for the fairly high rates of hypoglycemia in studies involving insulin-only artificial pancreases. ${ }^{9,10}$ Given these considerations, it seems unlikely that automated, safe glucose control can be achieved without a counterregulatory hormone such as glucagon.

There are several substantive challenges to the further development of a dual-hormone artificial pancreas. First, all studies to date have been in highly supervised settings, with the longest studies lasting fewer than 3 days. ${ }^{7}$ With greater confidence in these devices, longer-term studies in more authentic outpatient settings are necessary. Second, glucagon is commercially available in lyophylized form, which becomes unstable in solution. Although glucagon retains biological activity for a few days, new formulations that remain reliably stable in pumps are needed.

To date, insulin-only and dual-hormone artificial pancreases have been developed in parallel, with disparate trial designs that do not allow comparisons of performance between algorithms. Insulin-only and dual-hormone approaches will need to be compared in head-to-head trials under actual home-use conditions to clarify their relative merits. In the meantime, both insulin-only and dual-hormone approaches will have to prove their mettle in progressively less regimented settings.

\section{References}

1. Diabetes Control and Complications Trial Research Group. The effect of intensive treatment of diabetes on the development and progression of long-term complications in insulin-dependen diabetes mellitus: The Diabetes Control and Complications Trial Research Group. N Engl J Med 1993;329:977-86.

2. Nathan DM, Cleary PA, Backlund J-YC, et al. Intensive diabetes treatment and cardiovascular disease in type 1 diabetes mellitus. N Engl J Med 2005;353:2643-53.

3. Nathan DM, Zinman B, Cleary PA, et al.; DCCT/EDIC Research Group. Modern day clinical course of type 1 diabetes mellitus after 30-years duration. The diabetes control and complications trial/epidemiology of diabetes interventions and complications (DCCT/EDIC) and Pittsburgh epidemiology of diabetes complications (EDC) experience 1983-2005. Arch Intern Med 2009;169:1307-16.

4. Barton FB, Rickels MR, Alejandro R, et al. Improvement in outcomes of clinical islet transplantation: 1999-2010. Diabetes Care 2012;35:1436-45

5. Haidar A, Legault L, Dallaire M, et al. Glucose-responsive insulin and glucagon delivery (dual-hormone artificial pancreas) in adults with type 1 diabetes: a randomized crossover controlled trial. CMAJ 2013;185:297-305.

6. El-Khatib FH, Russell SJ, Nathan DM, et al. A bi-hormonal closed-loop blood glucose control device for type 1 diabetes. Sci Transl Med 2010;2:27ra27.

7. Russell SJ, El-Khatib FH, Nathan DM, et al. Blood glucose control in type 1 diabetes with a bihormonal bionic endocrine pancreas. Diabetes Care 2012;35:2148-55.

8. Castle JR, Engle JM, El Youssef J, et al. Novel use of glucagon in a closed-loop system for prevention of hypoglycemia in type 1 diabetes. Diabetes Care 2010;33:1282-7.

9. Steil GM, Rebrin K, Darwin C, et al. Feasibility of automating insulin delivery for the treatment of type 1 diabetes. Diabetes 2006;55:3344-50

10. Weinzimer SA, Steil GM, Swan KL, et al. Fully automated closed-loop insulin delivery versus semiautomated hybrid control in pediatric patients with type 1 diabetes using an artificial pancreas. Diabetes Care 2008;31:934-9.

Affiliation: David Nathan and Steven Russell are with the Massachusetts General Hospital, Harvard Medical School, Boston, Mass.

Contributors: Both of the authors contributed substantively to the writing and editing of the manuscript, and approved the final version submitted for publication. 